\title{
A!
}

This is an electronic reprint of the original article.

This reprint may differ from the original in pagination and typographic detail.

\author{
Orponen, Pekka
}

\section{Design methods for 3D wireframe DNA nanostructures}

\section{Published in:}

Natural Computing

DOI:

10.1007/s11047-017-9647-9

Published: 01/03/2018

Document Version

Peer reviewed version

Please cite the original version:

Orponen, P. (2018). Design methods for 3D wireframe DNA nanostructures. Natural Computing, 17(1), 147-160. https://doi.org/10.1007/s11047-017-9647-9

This material is protected by copyright and other intellectual property rights, and duplication or sale of all or part of any of the repository collections is not permitted, except that material may be duplicated by you for your research use or educational purposes in electronic or print form. You must obtain permission for any other use. Electronic or print copies may not be offered, whether for sale or otherwise to anyone who is not an authorised user. 


\section{Design methods for 3D wireframe DNA nanostructures}

\section{Pekka Orponen $^{\dagger}$}

\begin{abstract}
The field of structural DNA nanotechnology aims at the systematic development of self-assembling nanostructures using DNA as the construction material. Research in this area is progressing rapidly, and the controlled, computer-aided design of increasingly complex structures is becoming feasible. One thread of this endeavour is the design and characterisation of selfassembling 3D nanostructures based on wireframe polyhedral models. This article aims to illustrate some of the key developments in this direction, in sufficient detail so that the reader can achieve a general understanding of the main concepts and approaches. The emphasis is on the design principles rather than experimental methodology, and the role of computer science and computational tools is set forth.
\end{abstract}

Keywords DNA nanotechnology · DNA origami · Selfassembly · 3D nanostructures · Wireframe models

\section{Introduction and brief history}

The powerful idea of using synthetic DNA to create geometrically well-characterised nanostructures was first introduced by Nadrian Seeman in the early 1980's (Seeman 1981, 1982). Since then this conception has spawned the flourishing field of structural DNA nanotechnology (Pinheiro et al. 2011; Seeman 2015), which now has an increasing number of applications in biochemical and biomedical research, as well as in several areas of nanoscience and -technology (Seeman 2010; Krishnan and Simmel 2011; Zhang et al. 2014; Chandrasekaran et al. 2016a; Linko et al. 2016). Seeman's original main motivation was in creating regular threedimensional DNA lattices that could be used as frameworks to position macromolecules in a spatially periodic arrangement for further uses. Crystallographic studies of proteins were suggested in (Seeman 1982), but later proposed applications include e.g. artificial catalysts (Zhao et al. 2016) and plasmonic devices (Kuzyk et al. 2012; Tian et al. 2015; Gür et al. 2016). In addition to 3D lattices, also lattices in two dimensions and finite polyhedra were suggested in (Seeman 1982).

The immediate follow-up to Seeman's early works considered the detailed design and characterisation of the immobile Holliday junctions that were proposed in

\footnotetext{
+ Department of Computer Science, Aalto University, Fl-00076 Aalto, Finland. E-mail: pekka.orponen@aalto.fi

${ }^{1}$ We use the standard abbreviations ssDNA = single-stranded DNA, dsDNA = double-stranded DNA.
}

(Seeman 1981, 1982) as the fundamental building blocks of the envisioned DNA lattices (Seeman and Kallenbach 1983; Kallenbach et al. 1983; Cooper and Hagerman 1987). Then in 1991, Chen and Seeman presented the first detailed design of a DNA polyhedron, a cube composed by ligating and hybridising together ten synthetic ssDNA strands (Chen and Seeman 1991). This work was also the first to be accompanied by a (partial) experimental characterisation of the designed structure. A design and partial characterisation of a truncated DNA octahedron, based on a somewhat different approach, was presented three years later in (Zhang and Seeman 1994), and a design (not synthesised) for a regular octahedron was proposed in ( $\mathrm{Li}$ et al. 1996).

A new approach towards the development of 3D DNA polyhedra was introduced by Shih, Quispe and Joyce in 2004, when they presented a design for a regular octahedron that was based on folding a single synthetic long ssDNA ${ }^{1}$ strand upon itself (Shih et al. 2004), using as vertex motifs the four-way junctions from (Seeman 1981, 1982) and as edge motifs the DX and PX crossover schemes from (Fu and Seeman 1993; Seeman 2001). The resulting molecular complex was experimentally characterised by means of both gel-shift analysis and cryo-electron microscopy.

Two years later, Paul Rothemund simplified and generalised the single-strand folding approach remarkably by his idea of DNA origami (Rothemund 2006a). This powerful technique is based on folding a generic long scaffold strand, commonly the 7,249 $\mathrm{nt}^{2}$ ssDNA genome of bacteriophage M13mp18, to the desired target shape with the help of short, 20-50 nt, shape-specific staple strands. Rothemund's seminal publication demonstrated the versatility of this technique by a number of impressive 2D designs, and has since then led to a large number of further developments and applications. In particular, a number of 3D extensions of the approach have been demonstrated, some of which will be reviewed here in more detail.

Several of the earliest 3D origami designs, e.g. (Ke et al. 2009; Dietz et al. 2009; Han et al. 2011), were based on volume- or face-filling packings of double helices, ${ }^{3}$ but in the present overview we shall focus exclusively on wireframe-type designs, where the desired shape is formed

\footnotetext{
${ }^{2} \mathrm{nt}=$ nucleotide.

${ }^{3}$ An interesting non-origami approach to volume-filling 3D designs, using DNA "bricks", is presented in (Ke et al. 2012).
} 
by an open mesh of double-helix edges. This family of designs can be further conceptually partitioned into "braided" (non-origami) designs, where the mesh is composed by entwining together a number of elementary motifs (Chen and Seeman 1991; Zhang and Seeman 1994; Goodman et al. 2004; Shih et al. 2004; Goodman et al. 2005; He et al. 2008; Zhang et al. 2008), "modular" origami designs, where the mesh is built up from distinct elementary DNA origami components (Rothemund 2006b; Douglas et al. 2009; linuma et al. 2014), and "global" origami designs, where the origami scaffold strand is first routed according to the graph-theoretic structure of the mesh and then stapled together to achieve the desired 3D conformation (Han et al. 2013; Benson et al. 2015; Zhang et al. 2015; Veneziano et al. 2016).

In the following, we shall discuss some sample designs from each of these categories in roughly historical order, starting from the braided designs of (Chen and Seeman 1991; Shih et al. 2004; He et al. 2008) in Section 2, followed by the modular origami designs of (Rothemund 2006b; Douglas et al. 2009; linuma et al. 2014) in Section 3, and concluding with the global origami designs of (Han et al. 2013; Benson et al. 2015; Veneziano et al. 2016) in Section 4. In Section 5 we discuss briefly the role of computer science and computation in this research area, and conclude with some summary remarks in Section 6.

\section{Braided designs}

\subsection{Chen and Seeman, 1991}

The first detailed design for a 3D DNA polyhedron was the DNA cube presented by Chen and Seeman in (Nature, 1991). Their design comprises ten carefully crafted $80 \mathrm{nt}$ ssDNA strands (containing e.g. no repeated 6 nt segments) which are ligated and hybridised together as summarised in Fig. 1, eventually forming 12 dsDNA edges that bound the six faces of the cube.

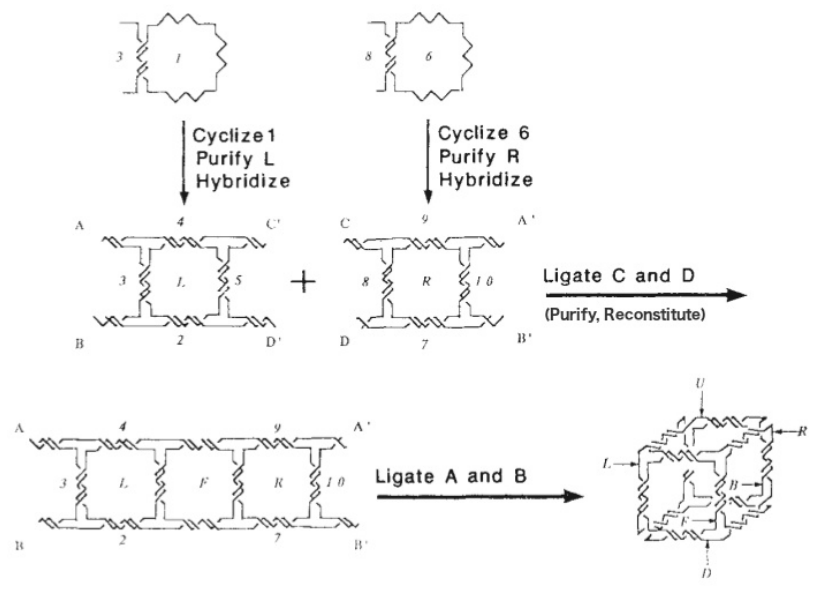

Fig. 1 Design of a DNA cube by braiding together ten ssDNA strands. Reprinted with permission from (Chen and Seeman 1991)

In the first step (Fig. 1, rows 1-2), linear ssDNA strands 1 and 6 are (a) cyclised by ligation and (b) hybridised with linear strands 2-5 and 7-10, respectively, to form the Left $(L)$ and Right $(R)$ faces of the cube, with complementary sticky ends $A, B, C^{\prime}, D^{\prime}$ for $L$ and $A^{\prime}, B^{\prime}, C, D$ for $R$.

In the second step (Fig. 1, rows 2-3), the sticky-end pairs ( $C$, $\left.C^{\prime}\right)$ and $\left(D, D^{\prime}\right)$ are combined by ligation, joining the faces $L$ and $R$ and concomitantly also creating the front $(F)$ face of the cube. In two further steps (not shown in Fig. 1), the resulting $L-F-R$ complexes are purified and rehybridised, and the nicks between strand pairs $(4,9)$ and $(2,7)$ are sealed. In a final step (Fig. 1, row 3 ), sticky-end pairs $\left(A, A^{\prime}\right)$ and $\left(B, B^{\prime}\right)$ are ligated together, closing the design and creating a wireframe DNA structure with the connectivity of a cube.

The article (Chen and Seeman 1991) presents a gel analysis of each stage of this synthesis process, validating that the correct intermediate and final compounds are produced. However, since there is no characterisation of the geometry of the final molecular complex, the authors do not claim that it actually has the overall shape of a cube, only that it has the right connectivity. ${ }^{4}$

The synthesis protocol is relatively complicated, because it takes the approach of catenating together distinct circular ssDNA strands that constitute the faces of the cube, by a sophisticated sequence of hybridisations and ligations. Later techniques that use a single backbone or "scaffold" ssDNA strand that runs through the whole structure, or focus on the vertex rather than the face motifs, do not create catenanes and hence achieve similar results with

other convex polyhedron with only triangular faces would not have this problem (Cromwell 1999, Chapter 6). ${ }^{4}$ In fact, a cube is not an ideal test structure for DNA polyhedral designs,
because unless the corner joints are stiff, a wireframe cube is not
structurally rigid, i.e. in a rod-hinge model it flexes. A tetrahedron or any 
much simpler protocols, ideally even in a single-pot reaction.

A further problem identified in this early approach is the need for detailed stoichiometry of the component strands. If the proportions of the reactants are not exactly right, then incomplete structures form, and purification may be difficult because the sizes of the correct and incorrect structures are close to each other (Rothemund 2006b).

\subsection{Shih et al., 2004}

An innovative alternative approach to the creation of polyhedral DNA structures was pioneered by Shih, Quispe and Joyce in (Shih et al. 2004). Instead of composing the target structure from shorter strands in a multi-stage process, these authors propose folding it from a single long strand, joined by a small number of helper strands, in a one-pot synthesis. Some of the advantages of this approach are that (a) the synthesis can be completed in a single mixing and cooling process, (b) since the backbone of the structure is made up of a single strand, stoichiometry is not a concern, and (c) since the strand folds (in a successful design) from its denatured state into the eventual structure without any topological or kinetic traps, the structures could in principle be mass-produced by cloning the requisite DNA sequences.

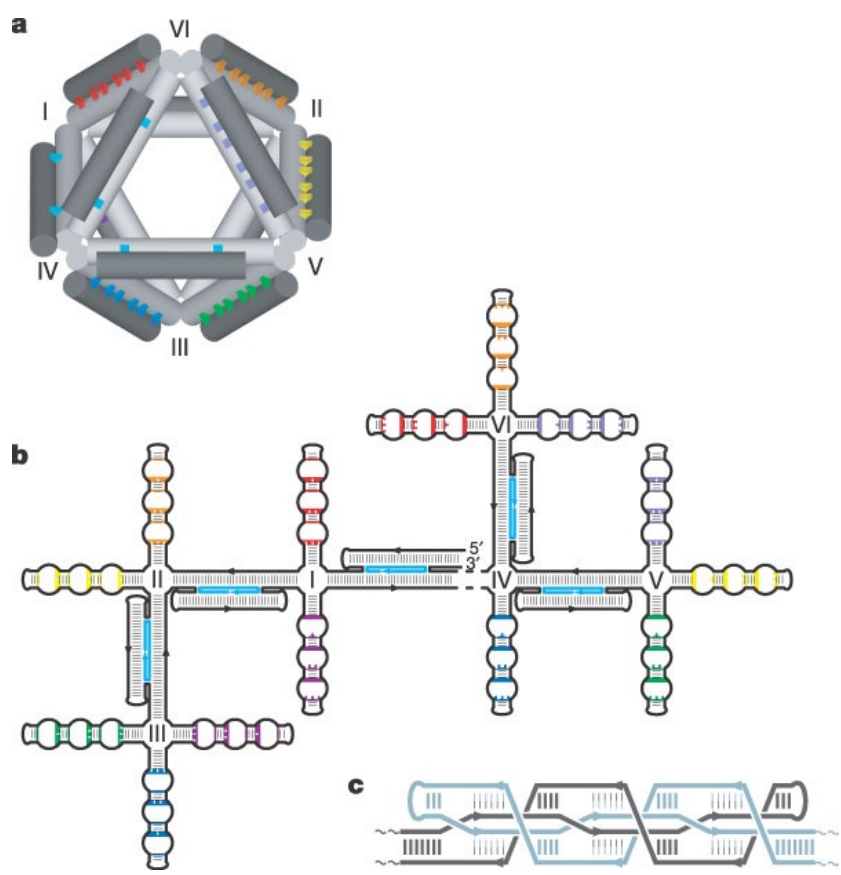

Fig. 2 Folding of a DNA octahedron from a single long ssDNA strand. Reprinted with permission from (Shih et al. 2004)

As a demonstration of this idea, the article (Shih et al. 2004) presents the folding of a regular octahedron from a synthetic 1,669 nt ssDNA backbone strand, augmented by five 40 nt "helper" strands (Fig. 2). The design builds in an innovative way on a number of motifs introduced earlier. The six vertices of the octahedron follow the four-way junction design from (Seeman 1981, 1982), and of the 12 edges, five are constituted according to the two-helix double-crossover (DX) scheme from (Fu and Seeman 1993) and seven according to the paranemic crossover (PX) scheme from (Seeman 2001). Each edge of the structure thus comprises two interleaved double-helical domains of DNA. (In Fig. 2(a) and Fig.2(b), the DX-based edge structures or "struts" are indicated in cyan blue and the PXbased struts in rainbow colours. Fig. 2(c) presents the crossover schematic of a PX strut.) The five helper strands partake in the forming of the DX-based struts. The nucleotide sequences for the backbone and the helper strands are carefully designed to form the correct secondary structures and to minimise the possibility of spurious hybridisations.

The folding of the structure proceeds in two phases. In the first phase, the five helper strands hybridise with their respective complementary domains in the backbone to form the five DX-based struts, and the rest of the backbone folds upon itself to form fourteen $76 \mathrm{nt}$ loops, each corresponding to one half of an eventual PX-based strut (Fig. 2(b)). In the second phase, the backbone loops hybridise in pairs to form the seven PX struts, resulting in the structure of Fig. 2(a). In Fig. 2(b) the PX pairings are indicated by matching colours of the strand loops.
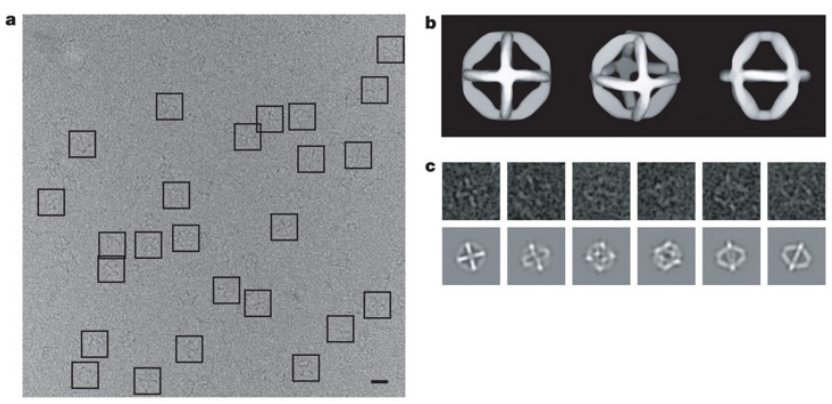

Fig. 3 Visualisation of the DNA octahedron based on cryo-electron microscopy. Scale bar $20 \mathrm{~nm}$. Reprinted with permission from (Shih et al. 2004)

From gel mobility-shift data, the yield of correctly folded structures was estimated at approximately $50 \%$ (Shih et al. 2004), and the geometry of the resulting molecular complexes was characterised by cryo-electron microscopy. Fig. 3 presents a summary of this characterisation: (a) raw micrograph, with images of individual structures boxed, (b) single-particle reconstruction, based on a large sample of individual images and imposed symmetry assumptions, (c) correspondences of six raw images with the reconstructed structure. 
As a design method, this single-strand folding approach would generalise also to other polyhedra, contingent on the availability of appropriate degree- $k$ vertex motifs, and limited by the constraints on designing appropriate length versions of the DX and PX motifs. These concerns notwithstanding, the general approach - formulated in graph-theoretic terms - would be to consider the graph $G=(V, E)$ representing the vertices $V$ and edges $E$ of the polyhedron of interest, and select some spanning tree $T \subseteq$ $E$ for it. ${ }^{5}$ Then the edges $e \in T$ comprising the spanning tree would be represented as DX motifs, and the edges $e \in$ $E \backslash T$ complementing the spanning tree into the full polyhedron as PX motifs.

To what extent this general procedure for creating 3D wireframe structures would work experimentally remains largely unexplored, because the method requires detailed control of the nucleotide sequence of the backbone strand, and synthesising designer strands on the order of thousands of nucleotides has at least so far been expensive and error-prone. Hence the technique was largely superseded by the introduction of Rothemund's DNA origami method (Rothemund 2006a), which only requires the scaffold strand to be (sufficiently close to) random, and moreover enables the use of a single generic scaffold strand for a great variety of designs. However, recent progress in the synthesis of genome-length strands (Gibson et al. 2009, 2010; Hughes and Ellington 2017) may eventually provide an opportunity to revisit also the singlestrand folding framework. ${ }^{6}$

\subsection{He et al., 2008}

A rather different approach to the synthesis of wireframe structures was reported in the article (He et al. 2008). Based on experience from earlier work with 2D structures (He et al. 2005), the authors surmised that by adjusting the structural flexibility of elementary motifs and their concentration in a one-pot assembly process, one could promote the spontaneous formation of higher-order structures of a desired type. In the work reported in (He et al. 2008), basic three-point-star (tri-star) motifs with stickyend terminals were made to self-assemble into tetrahedra, dodecahedra and truncated icosahedra (buckyballs), depending on the number of unpaired bases at the centre of the tri-star motif and the DNA concentration in solution.

\footnotetext{
${ }^{5}$ A spanning tree of a graph $G$ is a cycle-free set of edges that connects all vertices of $G$ (Diestel 2017, Section 1.5). Efficient algorithms for finding spanning trees exist and are in wide use (Cormen et al. 2009, Chapter 23). ${ }^{6}$ It is noted in passing at the end of (Shih et al. 2004) that their design could be simplified further, with some loss of rigidity, by replacing the DX struts by simple duplex struts, and the PX struts by hairpin loops that are first cleaved open by restriction enzymes and then joined by ligation to
}

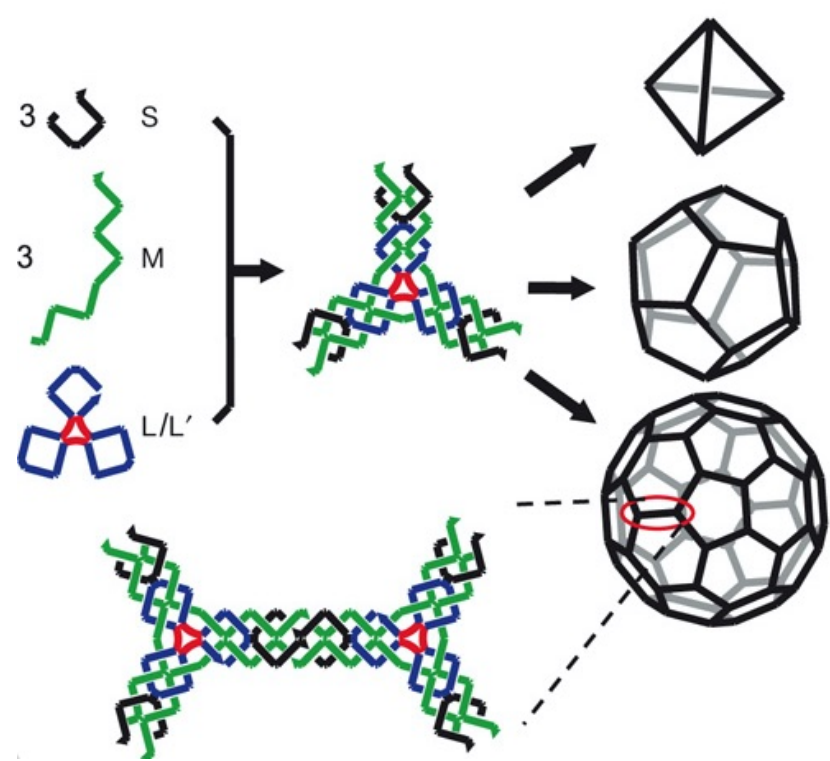

Fig. 4 Schemata for self-assembling tri-star motifs into polyhedral structures. Reprinted with permission from (He et al. 2008)

The basic tri-star motif used in (He et al. 2005, 2008) is presented in Fig. 4. It can be seen as a three-armed generalisation of the DX double-crossover scheme from (Fu and Seeman 1993) and consists of a long repetitive central strand $L$ (blue+red), three identical medium-length strands $M$ (green) that pass through the motif by crossing from one arm to another, and three identical short strands $S$ (black) that complement the $M$ strands at the terminal ends of the arms. The central strand $L$ contains three short singlestranded segments or "loops" (red) whose length has an important effect on the outcome of the self-assembly process. Adding bases to the central loops increases the flexibility of the motif and its amenability to sharper spatial angles.

The folding of a target polyhedron proceeds in two stages: first the individual $L, M$ and $S$ strands coalesce into tri-star motifs, and in the second stage these hybridise at their terminal sticky ends to form the polyhedral complex. According to the experimental data presented in (He et al. 2008), the outcome of the second stage depends on the length of the central loops in the $L$ strands and the concentration of DNA in the solution: short central loops impose obtuse angles, and high DNA concentration favours large assemblies. At central loop lengths of $5 \mathrm{nt}$ each, tetrahedral complexes were observed to form when the DNA strand concentration was $<100 \mathrm{nM}$, whereas at

their partners. One of the reviewers of the present survey also asked if the design could be based on DX-type struts alone. From the strand routing point of view this would seem to be possible, however with the backbone strand crossing itself at some point(s), so that one would need to take care also that the planned routing does not form a knot (cf. Section 4.2). The folding of this type of design has most likely not been experimentally tested. 
central loop lengths of $3 \mathrm{nt}$, the outcome was dodecahedra at low DNA concentration ( $50 \mathrm{nM}$ ) and buckyballs at high DNA concentration ( $500 \mathrm{nM}$ ). From gel-shift data, the yield for tetrahedra was estimated at $90 \%$, for dodecahedra at $76 \%$ and for buckyballs at $69 \%$.

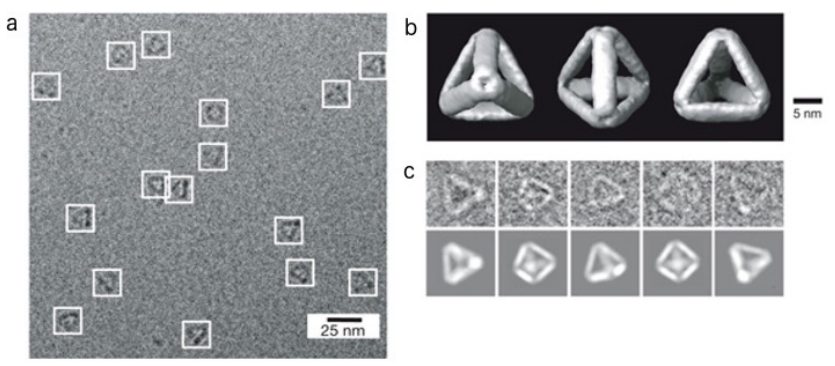

Fig. 5 Visualisation of self-assembled tetrahedral structures based on cryo-electron microscopy. Reprinted with permission from (He et al. 2008)

Fig. 5 presents a cryo-electron microscopy characterisation of the geometry of the tetrahedral complexes (imaging data for the other structures is available in the original publication): (a) raw micrograph, (b) single-particle reconstruction, (c) correspondences of five raw images with the reconstructed structure.

Along the same research line, article (Zhang et al. 2008) reports on applying a five-point-star motif to synthesise DNA icosahedra and large nanocages. The advantage of this methodology is that it achieves one-pot, high-yield self-assembly of well-characterised structures from very simple designs and minimal control mechanisms. Its limitation, on the other hand, is that because of the symmetric nature of the synthesis process, it is only applicable to highly regular structures and does not seem to provide a pathway to irregular ones.

\section{Modular origami designs}

The landscape of DNA nanostructure design changed with the introduction of Rothemund's DNA origami technique (Rothemund 2006a), which made possible the generalpurpose, computer-aided design of structures of almost arbitrary complexity, limited mainly by the length of the available scaffold strand. Because of the simplicity, robustness and generality of the procedure, most subsequent research on DNA nanostructures has been drawn to applications and extensions of this method, with alternative approaches to some extent having been neglected.

We shall not review the basic DNA origami technique here, because it has been amply covered in other sources, but shall move directly to its applications in the setting of 3D wireframe structures. In this section, we discuss approaches where the origami scaffolding is used to connect together elementary motifs, and in the next section we cover methods where the target structure emerges from a global routing of the scaffold strand along the edges of the wireframe mesh.

\subsection{Rothemund, 2006}

In a very interesting Festschrift paper (Rothemund 2006b), contemporaneous to the seminal DNA origami publication, Rothemund discusses polygonal network schemes deriving from Seeman's work, and proposes a new modular design approach based on connecting tri-star (and by extension also $k$-star) motifs with an origami-type scaffold strand (Fig. 6). The viability of this scheme has apparently never been tested in the laboratory, although some of the more recent approaches (Zhang et al. 2015; Veneziano et al. 2016) come close to the same result from a different direction.

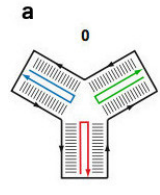

b

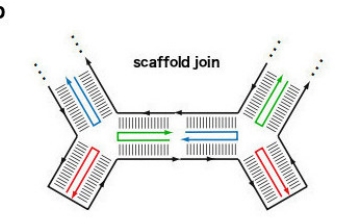

c

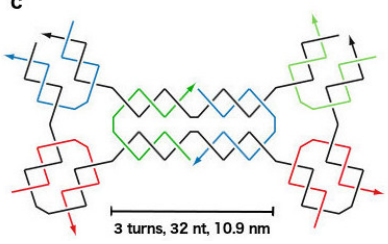

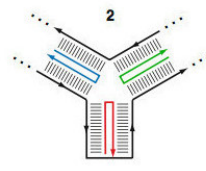
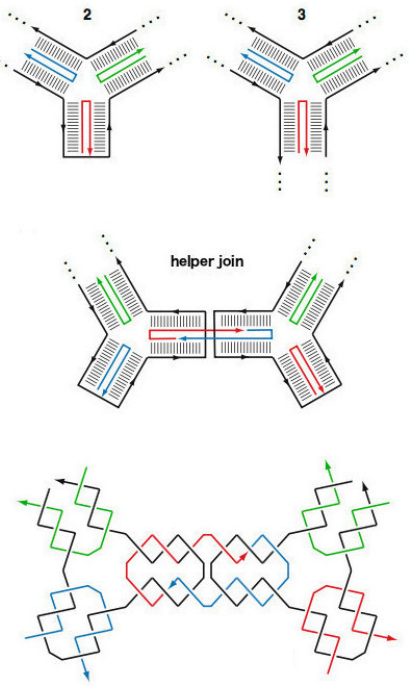

Fig. 6 (a) Tri-star motifs with 0-3 open ends. (b) Schematics for scaffold and helper joins. (c) Join details. Reprinted with permission from (Rothemund 2006b)

The basic building blocks of the proposed approach are four tri-star motifs with 0, 1, 2 or 3 "open" (unpaired) terminal ends, presented in Fig. 6(a). A tri-star with $i$ open terminals is said to be of "Type $i$ ". To achieve the DNA selfassembly design of a given trivalent simplicial polyhedron $P,{ }^{7}$ the polyhedron is first represented in terms of its Schlegel diagram $G_{P}$, which is a planar graph delineating the vertex-edge relationships of $P$. (Intuitively, one can obtain $G_{P}$ by "pulling $P$ flat" from one of its faces, so that the chosen face becomes the outer boundary of $G_{P}$.) Many

\footnotetext{
${ }^{7}$ A polyhedron is simplicial if it is homeomorphic (i.e. inflatable to) a sphere. It is trivalent if all vertices have exactly three neighbours.
} 
interesting polyhedra, e.g. all Platonic solids except the octahedron and the icosahedron, are trivalent, and this property is of course reflected also in their Schlegel diagrams. Fig. 7 depicts the trivalent Schlegel diagrams of the dodecahedron (top) and the buckyball (bottom).
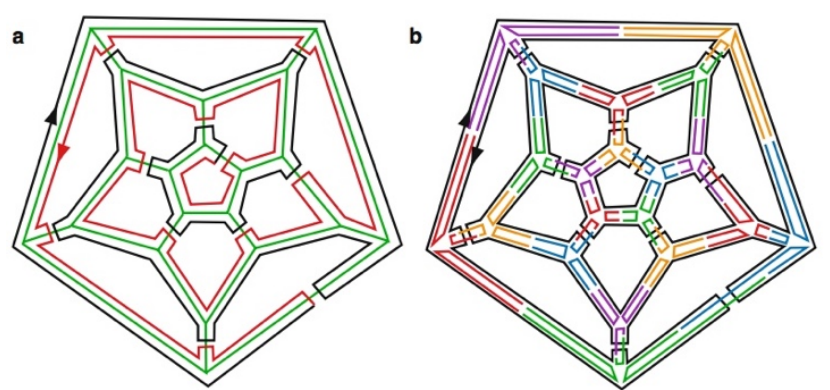

c

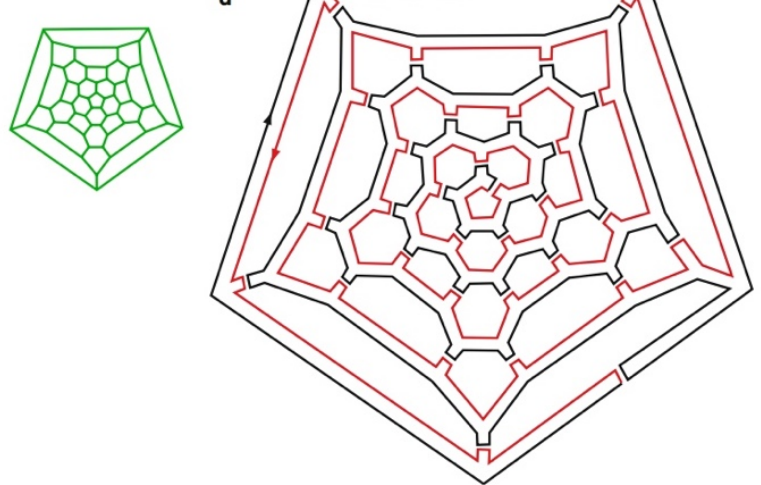

Fig. 7 Designs for a dodecahedron and a buckyball based on combining tri-star elements by scaffold and helper joins. Reprinted with permission from (Rothemund 2006b)

The design then proceeds by covering the vertices of $G_{P}$ by tri-star elements one by one, in the following steps:

1. A tri-star of Type 0 is placed on some arbitrarily chosen initial vertex. The outer boundary of this tri-star constitutes the initial scaffold strand loop.

2. A given partial covering is extended by:

a. choosing an uncovered neighbour vertex of some already placed tri-star $t$,

b. placing a tri-star $t^{\prime}$ of Type 0 on the new vertex,

c. "opening up" the terminal ends of $t$ and $t^{\prime}$ abutting each other and connecting the two tristars by a "scaffold join" pairing (Fig. 6(b)-(c), left). Thus, if $t$ was previously of Type $i$ and $t^{\prime}$ of Type 0 , now $t$ is of Type $i+1$ and $t^{\prime}$ is of Type 1 .

Step 2 is repeated until all the vertices have been covered.

\footnotetext{
${ }^{8}$ This specific connection is recognised and summarised in (Rothemund 2006b) with elegant conciseness: "William Shih has observed that singlestranded origami may be used to create arbitrary polygonal networks. To
}

Note that throughout this extension process, (i) the tri-star covered part of $G_{P}$ stays connected, (ii) all the terminals of the tri-star cover are "closed" and only opened one by one when a new tri-star is added, (iii) the scaffold strand loop, which is initialised in Step 1 and extended in each repeat of Step 2, stays connected. The eventual outcome of this process is thus a routing of the scaffold strand around the vertices of $G_{P}$, with "gaps" between neighbouring, but not scaffold-paired tri-star terminals (cf. Fig. 7). To complete the design, the gaps are finally closed by "helper join" pairings using the non-scaffold strands in the tri-star arms (Fig. 6(b)-(c), right).

Example designs for the dodecahedron and buckyball graphs following this scheme are presented in Fig. 7, where the part of the scaffold strand traversing "inwards" in the Schlegel diagram is indicated with black and the part traversing "outwards" is indicated with red. (The scaffoldconnected tri-star arms are extended to varying lengths in these schematics to make planar representation possible. In the actual 3D folding the tri-star motifs would be symmetric. Note also that the directions "inwards" and "outwards" in the planar Schlegel diagram correspond to "away from the viewer" and "towards the viewer" in 3D.) Also many other routings of the structure are possible, depending on the order in which the tri-star elements are placed on the vertices of the diagram in Step 2 of the design process.

A complementary point of view to this design scheme, not explicitly mentioned in (Rothemund 2006b), is that the scaffold-connected edges in fact constitute a spanning tree $T$ of $G_{P}$, and the scaffold strand traverses "twice around the tree" similarly as in e.g. the well-known TSP approximation heuristic in computer science (Cormen et al. 2009, Section 35.2). One could thus alternatively summarise the approach by saying that one chooses an arbitrary spanning tree $T$ of $G_{P}=(V, E)$, routes the scaffold strand twice around $T$, and then creates the nonspanning tree edges $e \in E \backslash T$ by helper joins.

This general point of view also relates Rothemund's design approach to that of (Shih et al. 2004), which is similarly implicitly based on building a spanning tree of a polyhedral graph using one type of connectors (DX struts), and creating the non-spanning tree edges with another type (PX pairings). ${ }^{8}$

Rothemund finally points out and discusses the possibility of extending the design method from trivalent to arbitrary simplicial polyhedra, contingent on the availability of well-

see this, replace helper joins with paranemic cohesion motifs and scaffold joins with Shih's double-crossover struts in all the diagrams of this section." 
behaving $k$-star motifs. From the spanning-tree point of view it is easy to see that the method in fact generalises to all connected spatial graphs - not just polyhedra - with a minimum degree of at least 3 , but whether such designs would actually fold in the laboratory remains open to experiment.

\subsection{Douglas et al., 2009}

Possibly the first laboratory-tested approach to creating 3D wireframe nanostructures based on the origami technique was presented in (Douglas et al. 2009). The article is primarily concerned with 3D structures constructed by a helix-packing method, but it outlines also the synthesis of a wireframe icosahedron composed by hybridising together three 2D origami -based flexible double-triangles (Fig. 8).

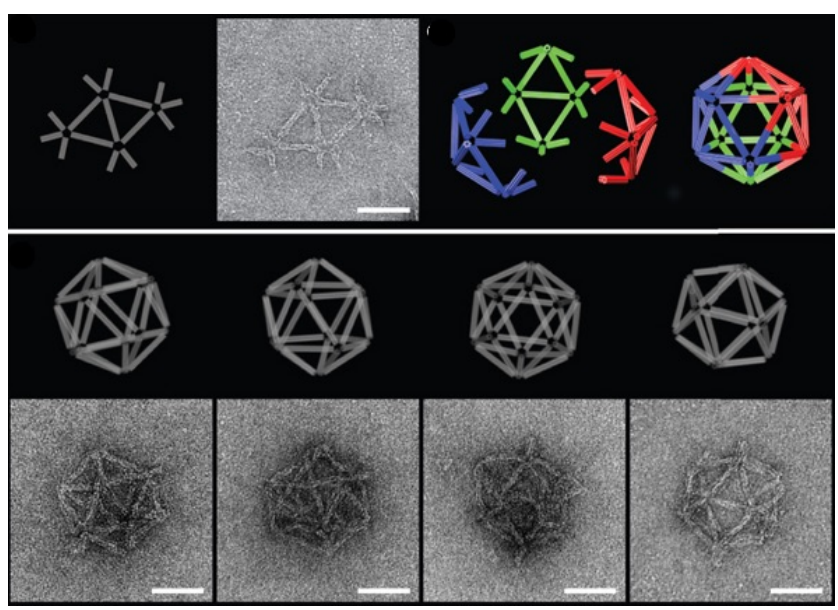

Fig. 8 Design and characterisation of a modular DNA origami icosahedron. Scale bars $100 \mathrm{~nm}$. Reprinted with permission from (Douglas et al. 2009)

The synthesis of the icosahedron is a two-stage process. In the first stage, each of the three component doubletriangles (Fig. 8, top left) is folded from an 8,100 nt scaffold strand, a handcrafted variant of the commonly used M13mp18 strand. To form these double-triangular structures, the scaffold strand is initially stapled into a branching tree of four 5-star motif vertices, connected by three scaffold join edges (each of which is in fact a six-helix bundle). Sticky-end strands at the ends of the half-strut long arms (which are again six-helix bundles) of the vertex motifs are designed so that two pairs of the arms hybridise, in order create the remaining two edges in the doubletriangle structure. Fig. 8 (top middle) presents a TEM image of one such complex.

Even though the double-triangles are geometrically similar and based on the same scaffold strand, they are made chemically different by using different cyclic permutations of the scaffold in each double-triangle. In the second stage of the process, the three component double-triangles are mixed together, and by a designed inter-component stickyend hybridisation of the remaining ten unpaired half-strut arms in each of the structures, the target icosahedron is created (Fig. 8, top right). The two lower rows of Fig. 8 present orthographic projection models and TEM images of four icosahedral complexes synthesised by this method.

\section{3 linuma et al., 2014}

The work presented in (linuma et al. 2014) addresses the task of creating large (edge-length $\sim 100 \mathrm{~nm}$ ) regular wireframe structures using the DNA origami technique. The basic design motif is a "DNA tripod" (Fig. 9(b)): a vertex element consisting of three $50 \mathrm{~nm}$ long arms, each constituted as a stiff 16-helix bundle, and a triangular twohelix strut that can be used to control the internal angles between the arms. DNA tripod arms can be connected into polyhedral edges by sticky-end "connector" elements, several of which were tried out in (linuma et al. 2014), and by this approach all trivalent convex polyhedra with isometric edges are in principle constructible.

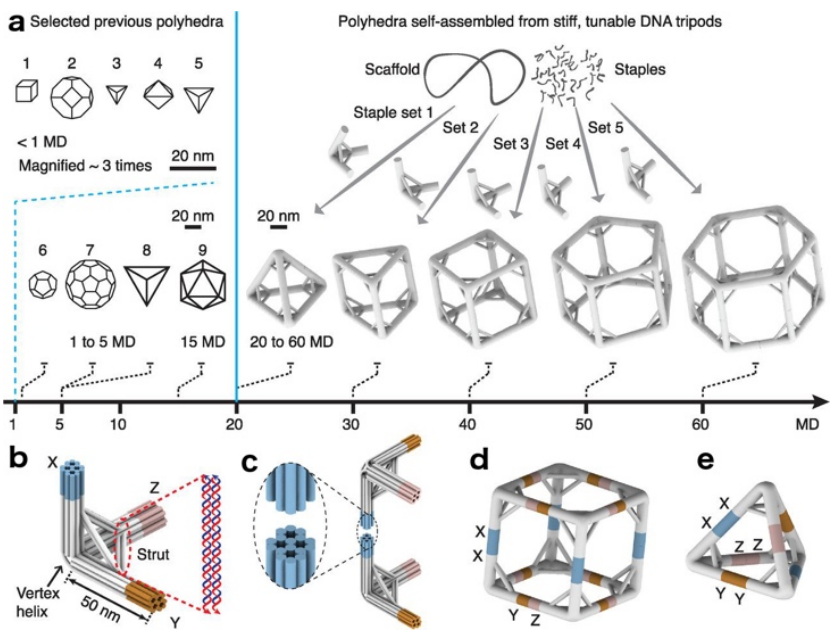

Fig. 9 The DNA tripod motif and polyhedral designs based on it. Reprinted with permission from (linuma et al. 2014)

The authors of (linuma et al. 2014) demonstrate this approach by synthesising the structures displayed in Fig. 9(a), which require adjusting the inter-edge angles between $60^{\circ}$ and $120^{\circ}$. The resulting complexes were imaged in solution, using the authors' newly-developed 3D super-resolution fluorescent microscopy technique "DNAPAINT".

\section{Global origami designs}

The methods presented in this section take a more "topdown" view of the design task than the previous ones. This is reflected both in the design methodology and in the complexity of the structures synthesised. 


\subsection{Han et al., 2013}

The article (Han et al. 2013) may be the first one to formulate the 3D wireframe DNA structure design task in terms of a global routing of the scaffold strand through a mesh framework, in such a way that the self-crossings of the scaffold form the vertices of the mesh. In this work, the mesh graph is not yet completely general, but is composed of square motifs (Fig. 10(a)), whose two sides are constituted by antiparallel segments of the scaffold strand (red), connected by staple strands that span the perimeters of the squares (grey). The helix arrangement around each square is carefully balanced so that the helical struts meet each other at a $90^{\circ}$ angle, rather than in a native $60^{\circ}$ angle conformation that each junction motif would take on its own because of intrinsic helix geometry (Fig. 10(b)-(e)). A typical scaffold routing path for a 2D DNA gridiron pattern is presented in Fig. 10(f), (g).

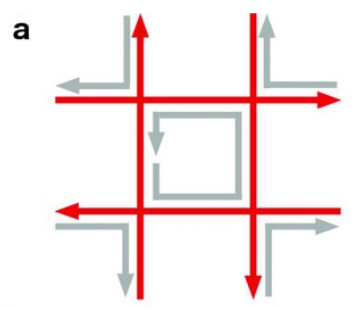

vs.
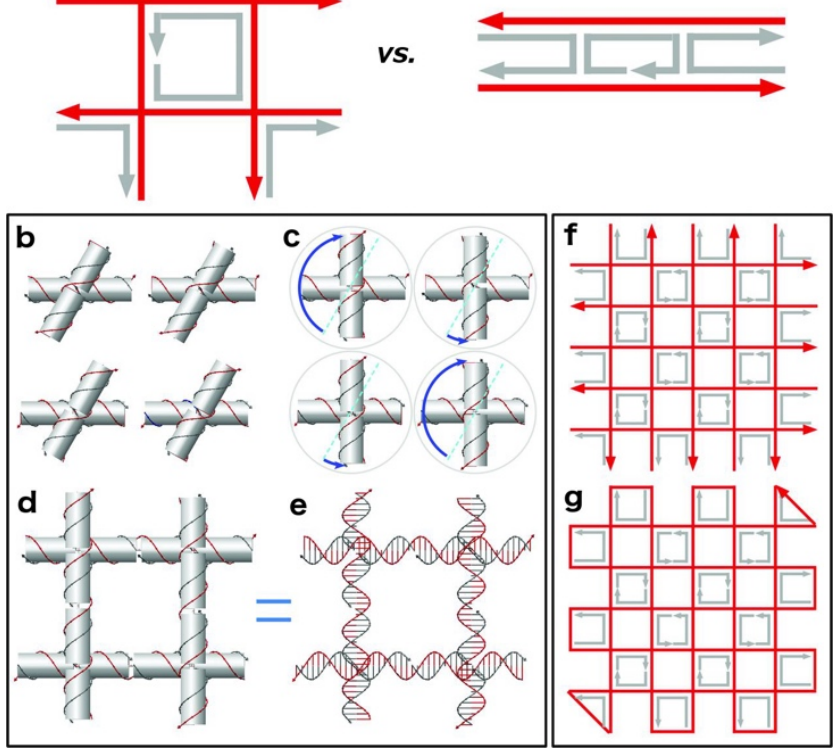

Fig. 10 The DNA gridiron design principles. Reprinted with permission from (Han et al. 2013)
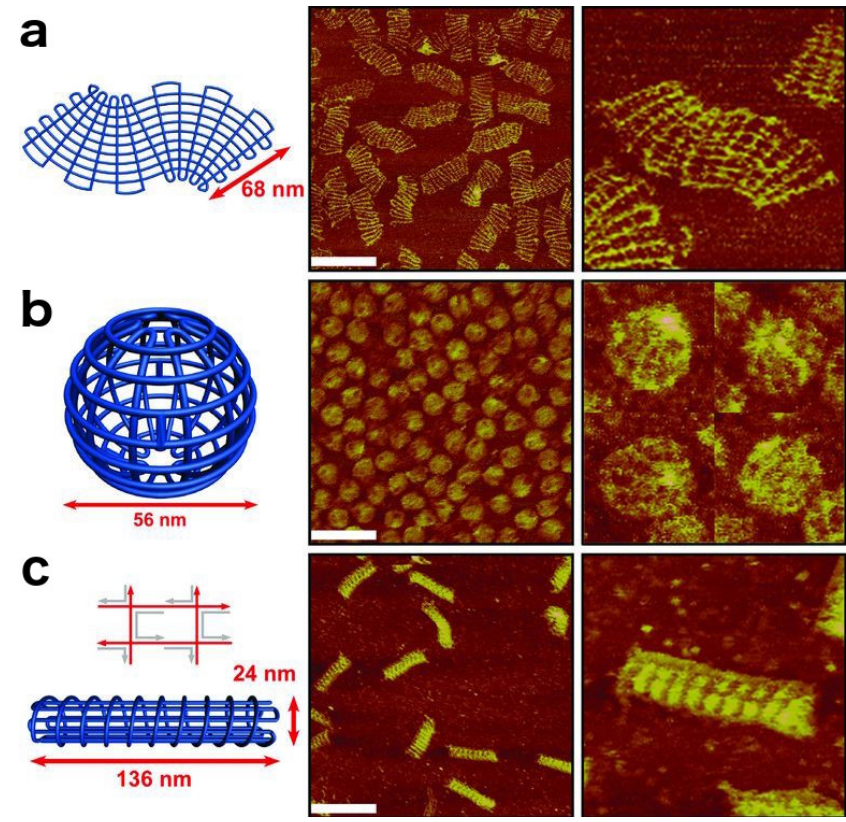

Fig. 11 Designs and AFM images of 2D and 3D structures synthesised as DNA gridiron meshes. Scale bars $200 \mathrm{~nm}$. Reprinted with permission from (Han et al. 2013)

The article (Han et al. 2013) focuses mostly on the design and synthesis of various 2D meshes, including ones with global curvature (Fig. 11(a)), but a number of 3D designs are presented too (Fig. 11(b), (c)). As regards 3D structures, the work exceeds in generality previous approaches, since by using different length DNA segments in different parts of the design, any convex 3D wireframe model composed of trapezoidal faces could in principle be rendered, possibly some nonconvex models too. ${ }^{9}$ A possible concern is that a mesh composed of trapezoidal elements is not necessarily structurally rigid (cf. Footnote 4); however this issue does not seem to be affecting the complexes displayed in the experimental data in (Han et al. 2013), possibly because of the tight intertwining of the crossing scaffold segments at the vertices of the mesh, which makes the vertices not hinge-like.

\subsection{Benson et al., 2015}

The article (Benson et al. 2015) presents a general topdown design methodology for rendering simplicial polyhedra as wireframe DNA origami structures. The starting point is the planar Schlegel diagram of a given polyhedron, and the goal is to route the scaffold strand along the edges of the diagram and staple it together at locations corresponding to the vertices, so that in selfassembly a molecular complex corresponding to the target polyhedron is created.

\footnotetext{
${ }^{9}$ Although the design methodologies in (Shih et al. 2004; Rothemund $2006 \mathrm{~b})$ are highly general too, this is not quite explicit in the articles and not reflected in the experimental work.
} 
A main difference to previous approaches is that there is no notion of composing the structure out of elementary vertex or edge motifs. Another difference in comparison to the technique in (Rothemund 2006b), which could also be seen as motif-less, is that all edges are scaffold-connected, i.e. there are no cross-scaffold helper joins, and to the technique in (Han et al. 2013) that the vertex degree of the polyhedron is not fixed, and moreover the mesh vertices are not created by crossings but rather by co-incident turns of the scaffold strand.
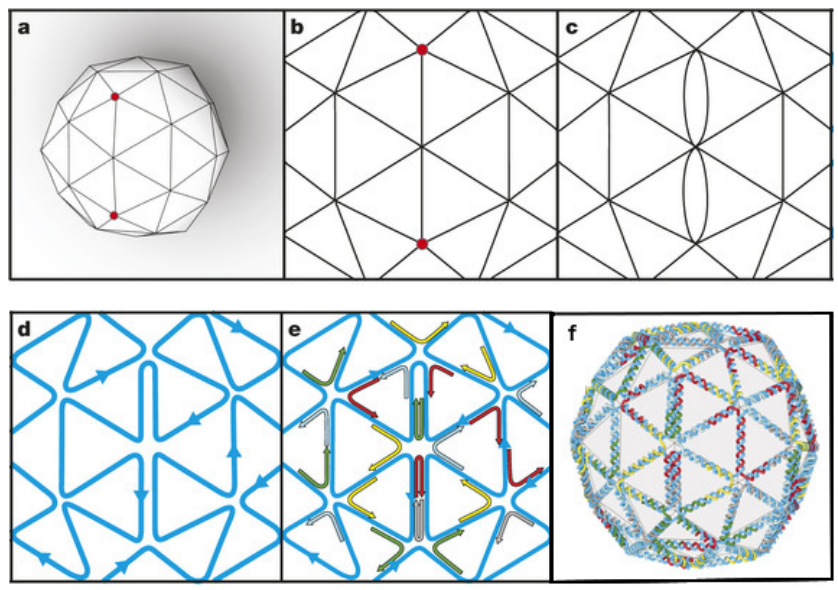

Fig. 12 A scaffold-routing based design process for the DNA rendering of a subdivided icosahedron. Reprinted with permission from (Benson et al. 2015)

Fig. 12 presents an outline of the design process for a sphere-like polyhedron $P$, technically a subdivided icosahedron or "dual buckyball" (Fig. 12(a)). A part of the Schlegel diagram $G_{P}$ of $P$ is shown in Fig. 12(b); ideally the circular scaffold strand would be routed so that it traces each of the edges in $G_{P}$ exactly once, thus forming an Eulerian circuit in the graph (Diestel 2017, Section 1.8). However, by a well-known theorem of Euler (1741; Diestel 2017, Section 1.8), this is possible if and only if all the vertices in $G_{P}$ are of even degree. Hence all the odd-degree vertices - of which there are always an even number (Diestel 2017, Section 1.2) - are matched pairwise by augmenting paths of auxiliary edges (Fig. 12(c)). The number of added edges is minimised by using the minimum-cost graph matching algorithm of Edmonds (1965).

In fact, the routing is aiming for a special type of Eulerian circuit which (i) does not cross itself at vertices, and (ii) even more strictly turns back, upon entering a vertex, along one of the two faces incident to the entering edge (left/right). An example of such an A-trail (Fleischner $1990)^{10}$ for $G_{P}$ is displayed in Fig. 12(d). One reason for this constraint is that one wishes to staple the segments of the

\footnotetext{
${ }^{10}$ The name A-trail presumably derives from the sharp "A-like" turns the path makes at each vertex.
}

scaffold together at each vertex as shown in Fig. 12(e), by staple strands that join two adjacent, but not consecutive segments of the scaffold. Another, and more fundamentally important reason is that an A-trail routing on a sphere is guaranteed to be unknotted, i.e. continuously deformable into a circle (and vice versa), and so creates no topological traps for the folding of the circular scaffold strand.

After an appropriate A-trail routing for the scaffold strand has been found, the detailed positions, lengths and phases of the DNA helices are optimised in order to minimise the strain created by the interhelix gaps at the vertex sites, where the scaffold strand needs to transit from one incident helix to the next. The eventual result is a strandlevel DNA model of the target polyhedron (Fig. 12(f)), from which the requisite staple strand sequences can be computed, given the scaffold strand sequence. The design process, including staple strand sequence generation, has been automated in a tool called vHelix, ${ }^{11}$ a DNA nanostructure design plugin for the Autodesk Maya 3D computer graphics and modelling platform.

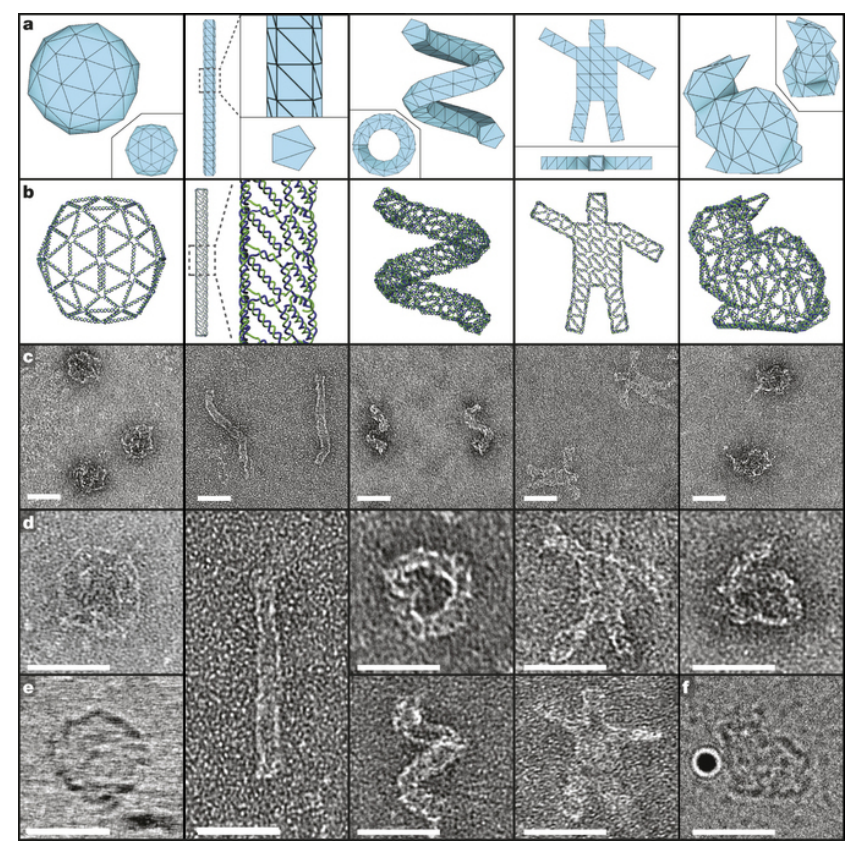

Fig. 13 Designs and images of simplicial polyhedra rendered as wireframe DNA origami meshes. Scale bars $50 \mathrm{~nm}$. Reprinted with permission from (Benson et al. 2015)

Fig. 13 presents a number of DNA polyhedra designed and synthesised using this approach. The first row shows the 3D mesh designs, the second row the strand-level DNA designs, and the three further rows electron micrographs of the resulting complexes. (The one furthest to the right is a variant of the "Stanford bunny", ${ }^{12}$ a widely-used 3D

\footnotetext{
${ }^{11}$ http://www.vhelix.net/

${ }^{12}$ https://en.wikipedia.org/wiki/Stanford_bunny
} 
computer graphics test model.) All imaging is from transmission electron microscopy, except for the sphere image at bottom left and the bunny image at bottom right, which are obtained by cryo-electron microscopy.

The target polyhedra are in this work generally assumed to be triangulated (have only triangular faces), which entails that their respective Schlegel diagrams are fully triangulated planar graphs. This is not strictly necessary for the method, but simplifies some considerations and guarantees that the wireframe model is structurally rigid (Cromwell 1999, Chapter 6).

It should also be noted that while finding ordinary Eulerian circuits in graphs is a standard task in computer science with a simple and efficient solution method (Cormen et al. 2009, Section 22), finding A-trails is an NP-complete problem. $^{13}$ In (Benson et al. 2015), a branch-and-bound search space exploration algorithm is developed for this task, and for the strain relaxation of the helix-level design, a physical springs-and-rods model is used.

According to (Benson et al. 2015), the DNA meshes presented in Fig. 13 in general fold well, with an average yield of $43 \%$. The highest reported yield of $92 \%$ was obtained for the subdivided icosahedron ( $n=42$ vertices, $m=120$ edges) and the lowest yield of $4.5 \%$ for the superficially simple "stickman" figure $(n=92, m=282)$. The visually complex "bunny" design $(n=70, m=204$ ) achieved an intermediate yield of $22 \%$. An additional benefit is that the folding seems to be stable also in lowsalt physiological buffers. A possible concern at least for some applications may be the floppiness of the singleduplex struts, although no systematic comparisons to e.g. DX-based struts have so far been made.

\subsection{Veneziano et al., 2016}

The recent article (Veneziano et al. 2016) presents another highly systematic and automated top-down approach to the DNA rendering of wireframe polyhedral structures. It is more closely connected to previously established design principles than the one in (Benson et al. 2015), and is in some ways more general, in others slightly more constrained than it. It will be interesting to see how the two approaches compare experimentally, and what new developments will emerge from them.

The starting point of the design method in (Veneziano et al. 2016) is again the Schlegel diagram $G_{P}=(V, E)$ of a given target polyhedron $P$ (Fig. 14), for which a spanning tree $T$

\footnotetext{
${ }^{13}$ More precisely, the problem is known to be NP-complete in Eulerian polyhedral graphs (Døvling Andersen and Fleischner 1995), but not in triangulated Eulerian polyhedral graphs. In fact, Fleischner (1990)
}

is first determined (Fig. 14(i)). Then the scaffold strand is routed twice around the spanning tree $T$ similarly as in (Rothemund 2006b; Fig. 14(ii)), and stapled together into double-duplex struts with DX-type connector motifs (Fig. 14(iii), 2). In addition to the spanning-tree edges $e \in T$, the struts also cover two opposing halves of each non-spanning tree edge $e^{\prime} \in E \backslash T$, and these are connected by stickyend helper join motifs to constitute these edges (Fig. 14(iii), $3)$. The vertices, which in this approach can in principle be of arbitrary degree, are reinforced by vertex staples, each of which spans either three or four adjacent strut stems (Fig. 14(iii), 1).

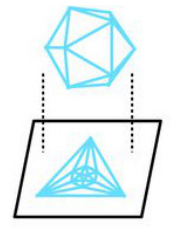

Schlegel diagram

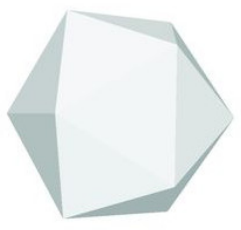

Target polyhedron

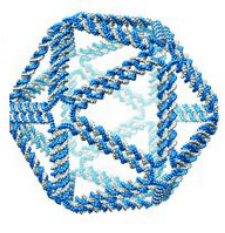

Atomic model
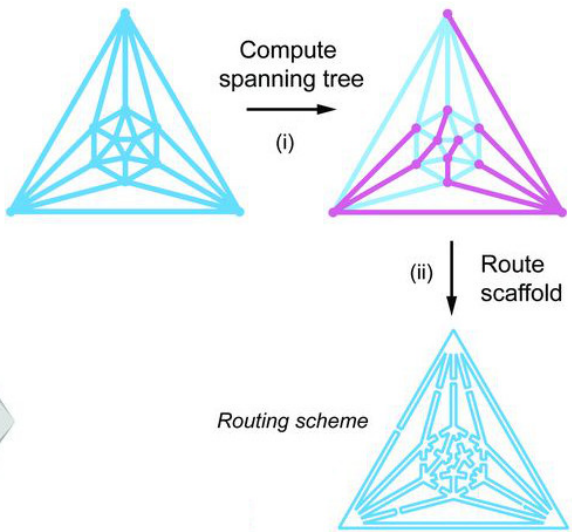

(iii) $\mid \begin{aligned} & \text { Assign } \\ & \text { staples }\end{aligned}$
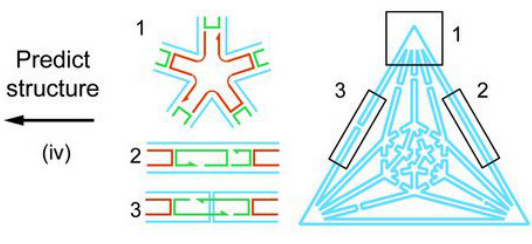

Sequence design (ii) $\mid \begin{aligned} & \text { Route } \\ & \text { scaffold }\end{aligned}$

Fig. 14 A spanning-tree routing based design process for wireframe DNA polyhedra. Reprinted with permission from (Veneziano et al. 2016)

Many of these design principles derive from a previous article by partly the same authors (Zhang et al. 2015), but (Veneziano et al. 2016) seems to be the first publication on 3D wireframe DNA structures that expresses the idea of spanning tree routings, augmented by helper join pairings to create the non-spanning tree edges, really explicitly and makes it the basis of a systematic, automated and empirically validated design method. The outcome is quite close to the designs proposed in (Rothemund 2006b), or indeed (Shih et al. 2004), but neither of these articles formulates their approach explicitly in terms of spanning tree routings. The idea is also presented briefly in (Benson et al. 2015, Extended Data Fig. 1), but only to compare the

conjectured that every triangulated Eulerian polyhedral graph has an Atrail, but even under this assumption, no polynomial time algorithm is known for finding such. 
A-trail routing method to previous design strategies. With respect to the apparently un-implemented proposal in (Rothemund 2006b), one should also note that although the results of the high-level designs in (Veneziano et al. 2016) are quite similar, the detailed edge and vertex motifs suggested in the two articles are rather different.

The design process described in (Veneziano et al. 2016) has been automated in the software tool DAEDALUS, ${ }^{14}$ and the article displays examples of a large number of symmetric DNA polyhedra designed using this tool. Experimental characterisations are presented for six of the polyhedra in the main article (three shown in Fig. 15), and in the supplementary material for a seventh. The reported yields for the designs are very high, with an average of $82 \%$ and a range between $60 \%$ (cube, $n=8, m=12$ ) and $93 \%$ (tetrahedron, $n=4, m=6$ ). Similarly as in (Benson et al. 2015), the structures fold well and are stable also in lowsalt physiological buffers.

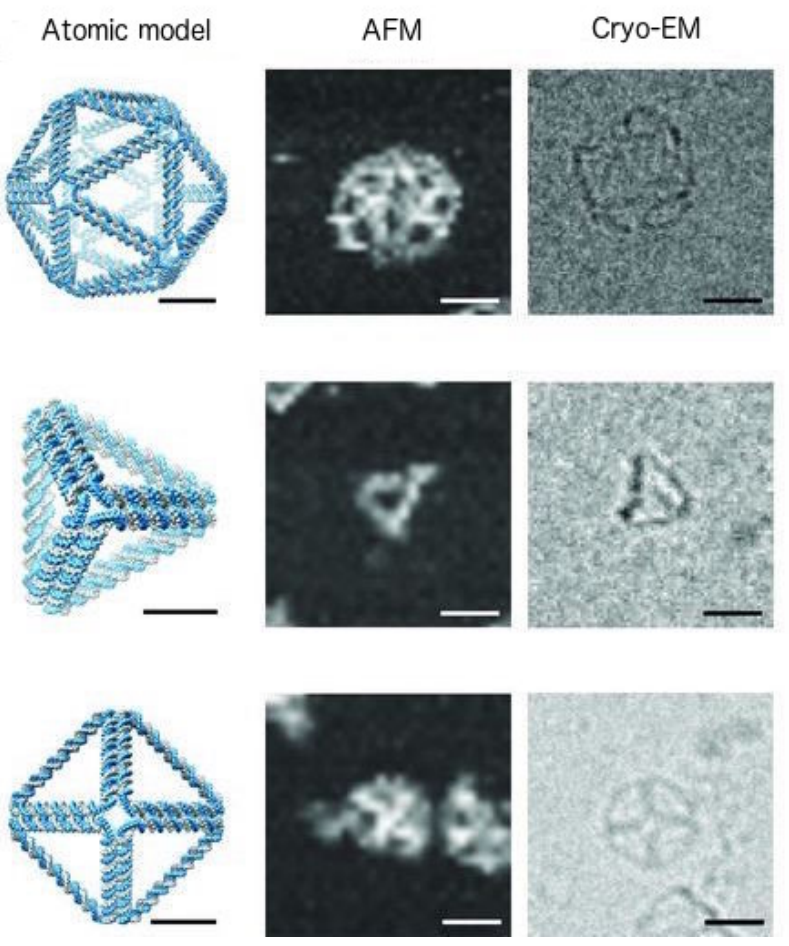

Fig. 15 Designs and images of spanning-tree routed wireframe DNA icosahedra, tetrahedra and octahedra. Scale bars $10 \mathrm{~nm}$ for atomic models, $20 \mathrm{~nm}$ for AFM and cryo-EM. Reprinted with permission from (Veneziano et al. 2016)

The advantages of the spanning-tree based design method in (Veneziano et al. 2016), as compared to the A-trail based method in (Benson et al. 2015), are that it is theoretically simpler and computationally more efficient (although the difference is probably not noticeable in practice), and it applies as such to arbitrary polyhedra, whereas generalising the A-trail based method to nonsimplicial polyhedra leads to quite nontrivial theoretical considerations. (However an extension to triangulated polyhedra on surfaces of any fixed genus was recently presented in (Mohammed and Hajij 2017)). A partial drawback of the spanning-tree method is that because of the double-routing of mesh edges, it uses twice the amount of scaffold DNA of the A-trail method, so that some of the complexes synthesised in (Benson et al. 2015, cf. also Fig. 13) would be unreachable with this approach using the standard M13mp18 scaffold strand. On the other hand, double-routing visibly increases the rigidity of the edges, and developments for longer scaffold strands are on the way: e.g. (Marchi et al. 2014) presents origami designs based on a biologically derived 51,466 nt scaffold strand and (Chandrasekaran et al. 2016b) surveys recent developments in this direction more broadly.

In its present form, the approach of (Veneziano et al. 2016) imposes some regularity on the achievable structures because of the internal design of the DNA struts (e.g. all edge lengths are required to be multiples of a full turn of DNA). But this is more of a compromise between the reliability and generality of the method, not an inherent constraint. A possible concern related to the scalability of the method is the relatively large number of cross-scaffold helper joins $(F-1$ for a simplicial polyhedron with $F$ faces), which could in complex designs lead to nonspecific interactions across the termini of unpaired half-edge struts. Considering the high yields of the syntheses reported in (Veneziano et al. 2016), this does not seem to be a problem at least for the structures shown in Fig. 15. These are relatively simple, however, so further experimentation with and development of the method is needed to expose more of its characteristics.

\section{The role of computer science and computation}

Computer science and computational methods impact the development of DNA nanotechnology, in the present case research on 3D wireframe DNA nanostructures, in at least four ways:

1. Computational tools are crucial for the design of increasingly complex structures and to implement high-level, generic design approaches. This aspect has been well recognised in the DNA nanotechnology community from the beginning, and particularly since the introduction of Rothemund's DNA origami technique. In the present context, in particular the Atrail based designs (Benson et al. 2015) could not have been achieved without computer support, and also in

\footnotetext{
${ }^{14}$ http://daedalus-dna-origami.org/
} 
the other approaches at least the detailed strand sequence listings are too laborious to be produced by hand. For non-origami schemes, an important and highly nontrivial computational task is also the design and optimisation of the nucleotide sequence so that it indeed folds into the desired conformation (Brenneman and Condon 2002; Dirks et al. 2004; Feldkamp and Niemeyer 2006).

2. Well packaged and easily accessible software is necessary for making the technology widely available outside of the core research community. This issue has also been well recognised, and in the domain of 3D wireframe structures, packages such as vHelix and DAEDALUS are aiming for maximally automated and user-friendly interfaces for the non-specialist users of the design tools. In other directions, e.g. the Cadnano ${ }^{15}$ software package is widely used for the design of helixpacked 3D DNA nanostructures, and the Mfold ${ }^{16}$ and NUPACK $^{17}$ packages for sequence design and secondary structure prediction tasks.

3. A computer science perspective brings clarity and generality to the planning of new design approaches. While nanostructure design is fundamentally rooted in the characteristics of the material, a high-level view can provide valuable complementary insights. In the present setting, this is illustrated for instance by the prevalence of the concept of spanning tree routings in several of the design approaches (Shih et al. 2004; Rothemund 2006b; Zhang et al. 2015; Veneziano et al. 2016), even though the concept was not explicitly recognised and fully utilised until (Veneziano et al. 2016). As another example, the A-trail based design technique (Benson et al. 2015) is deeply embedded in graph-theoretic concepts and algorithms. Further applications of graph-theoretic and topological ideas to the design of wireframe nanostructures are presented in (Klavžar and Rus 2013; Ellis-Monaghan et al. 2015, 2017; Mohammed and Hajij 2017; Morse et al. 2017).

4. Computational modelling and simulation tools will be increasingly useful to speed up the design process and reduce laboratory time and costs. As the complexity of the targeted structures increases, it becomes progressively more important to be able to pre-screen them computationally, before committing expensive laboratory resources to their experimental characterisation. In the context of 3D wireframe designs, important tools include e.g. the CanDo finiteelement modelling framework for DNA nanostructures, ${ }^{18}$ which is being integrated with the DAEDALUS design package, and the oxDNA molecular dynamics modelling framework, ${ }^{19}$ which has been used to simulate and visualise many interesting DNA structures and processes, including e.g. the A-trail based sphere ${ }^{20}$ and bunny ${ }^{21}$ molecular complexes. A closer integration of the design and simulation tools can be expected in the future, so that eventually automated, model-based pre-assessment of the structures can also be used to guide the design process.

\section{Summary}

Three-dimensional DNA nanostructure designs based on wireframe polyhedral models have evolved into an interesting alternative to the more established helixpacking 3D designs: several distinct approaches exist, and functionalisations are beginning to emerge. Wireframe designs are appealing both because they make more efficient use of the DNA scaffold than helix-packing approaches, and because they seem to fold with higher yield and remain more stable in low-salt, physiological buffer conditions.

Because of the inherent combinatorial complexity of wireframe designs, automation of the design process is a central task already for exploratory research, and even more so when aiming to make the methodology robust and generally available. Several computerised tools already exist for assisting in the high-level design stage, but these still need to be complemented by and integrated to numerical modelling software to help in validating and finetuning the designs before moving to the synthesis stage.

Acknowledgments Preparing this survey was made possible by a sabbatical leave from Aalto University, and most of it was written during stays at ETH Zürich (host Prof. Roger Wattenhofer), the University of Electro-Communications in Tokyo (host Prof. Shinnosuke Seki) and the Tokyo Institute of Technology (host Prof. Osamu Watanabe). I am very grateful for this opportunity. I also thank the two anonymous reviewers for their constructive and insightful comments.

\section{References}

Benson E, Mohammed A, Gardell J, et al (2015) DNA rendering of polyhedral meshes at the nanoscale. Nature 523:441-444. doi: 10.1038/nature14586

Brenneman A, Condon A (2002) Strand design for biomolecular computation. Nat Comput 287:3958. doi: 10.1016/S0304-3975(02)00135-4

\footnotetext{
${ }^{19}$ https://dna.physics.ox.ac.uk/

${ }^{20} \mathrm{http}: / /$ bit.ly/2djHZbU

${ }^{21}$ http://bit.ly/2dhxERD
}

\footnotetext{
${ }^{15} \mathrm{http}: / /$ cadnano.org/

${ }^{16} \mathrm{http}: / /$ unafold.rna.albany.edu/

${ }^{17}$ http://www.nupack.org/

${ }^{18}$ http://cando-dna-origami.org/
} 
Chandrasekaran AR, Anderson N, Kizer M, et al (2016a) Beyond the fold: Emerging biological applications of DNA origami. ChemBioChem 17:1081-1089. doi: 10.1002/cbic. 201600038

Chandrasekaran AR, Pushpanathan M, Halvorsen K (2016b) Evolution of DNA origami scaffolds. Mater Lett 170:221-224. doi: 10.1016/j.matlet.2016.01.161

Chen J, Seeman NC (1991) Synthesis from DNA of a molecule with the connectivity of a cube. Nature 350:631-633. doi: 10.1038/350631a0

Cooper JP, Hagerman PJ (1987) Gel electrophoretic analysis of the geometry of a DNA four-way junction. J Mol Biol 198:711-719.

Cormen TH, Leiserson CE, Rivest RL, Stein C (2009) Introduction to Algorithms, 3rd edn. MIT Press, Cambridge MA, USA

Cromwell PR (1999) Polyhedra. Cambridge University Press, Cambridge, UK

Diestel R (2017) Graph Theory, 5th edn. Springer-Verlag, Berlin Heidelberg, Germany

Dietz H, Douglas SM, Shih WM (2009) Folding DNA into twisted and curved nanoscale shapes. Science 325:725. doi: 10.1126/science. 1174251

Dirks RM, Lin M, Winfree E, Pierce NA (2004) Paradigms for computational nucleic acid design. Nucleic Acids Res 32:1392-1403. doi: 10.1093/nar/gkh291

Douglas SM, Dietz H, Liedl T, et al (2009) Self-assembly of DNA into nanoscale three-dimensional shapes. Nature 459:414-418. doi: 10.1038/nature08016

Døvling Andersen L, Fleischner H (1995) The NPcompleteness of finding A-trails in Eulerian graphs and of finding spanning trees in hypergraphs. Discrete Appl Math 59:203-214. doi: 10.1016/0166-218X(95)80001-K

Edmonds J (1965) Paths, trees, and flowers. Can J Math 17:449-467. doi: 10.4153/CJM-1965-045-4

Ellis-Monaghan JA, McDowell A, Moffatt I, Pangborn G (2015) DNA origami and the complexity of Eulerian circuits with turning costs. Nat Comput 14:491-503. doi: 10.1007/s11047-014-9457-2
Ellis-Monaghan JA, Pangborn G, Seeman NC, et al (2017) Design tools for reporter strands and DNA origami scaffold strands. Theor Comput Sci 671:69-78. doi: 10.1016/j.tcs.2016.10.007

Euler L (1741) Solutio problematis ad geometriam situs pertinentis. Comment Acad Sci Petropolitanae 8:128-140.

Feldkamp U, Niemeyer CM (2006) Rational design of DNA nanoarchitectures. Angew Chem Int Ed 45:18561876. doi: 10.1002/anie.200502358

Fleischner H (1990) Eulerian Graphs and Related Topics. Elsevier North Holland, Amsterdam

Fu TJ, Seeman NC (1993) DNA double-crossover molecules. Biochemistry (Mosc) 32:3211-3220. doi: 10.1021/bi00064a003

Gibson DG, Smith HO, Hutchison CA, et al (2010) Chemical synthesis of the mouse mitochondrial genome. Nat Meth 7:901-903. doi: 10.1038/nmeth.1515

Gibson DG, Young L, Chuang R-Y, et al (2009) Enzymatic assembly of DNA molecules up to several hundred kilobases. Nat Meth 6:343-345. doi: $10.1038 /$ nmeth. 1318

Goodman RP, Berry RM, Turberfield AJ (2004) The singlestep synthesis of a DNA tetrahedron. Chem Commun 1372-1373.

Goodman RP, Schaap IAT, Tardin CF, et al (2005) Rapid chiral assembly of rigid DNA building blocks for molecular nanofabrication. Science 310:1661. doi: 10.1126/science. 1120367

Gür FN, Schwarz FW, Ye J, et al (2016) Toward selfassembled plasmonic devices: High-yield arrangement of gold nanoparticles on DNA origami templates. ACS Nano 10:5374-5382. doi: 10.1021/acsnano.6b01537

Han D, Pal S, Nangreave J, et al (2011) DNA origami with complex curvatures in three-dimensional space. Science 332:342. doi: 10.1126/science.1202998

Han D, Pal S, Yang Y, et al (2013) DNA gridiron nanostructures based on four-arm junctions. Science 339:1412. doi: 10.1126/science.1232252

He Y, Chen Y, Liu H, et al (2005) Self-assembly of hexagonal DNA two-dimensional (2D) Arrays. J 
Am Chem Soc 127:12202-12203. doi:

10.1021/ja0541938

He Y, Ye T, Su M, et al (2008) Hierarchical self-assembly of DNA into symmetric supramolecular polyhedra. Nature 452:198-201. doi: 10.1038/nature06597

Hughes RA, Ellington AD (2017) Synthetic DNA synthesis and assembly: putting the synthetic in synthetic biology. Cold Spring Harb Perspect Biol. doi: 10.1101/cshperspect.a023812

linuma $R$, Ke $Y$, Jungmann $R$, et al (2014) Polyhedra selfassembled from DNA tripods and characterized with 3D DNA-PAINT. Science 344:65. doi: $10.1126 /$ science. 1250944

Kallenbach NR, Ma R-I, Seeman NC (1983) An immobile nucleic acid junction constructed from oligonucleotides. Nature 305:829-831. doi: $10.1038 / 305829 \mathrm{a} 0$

Ke Y, Ong LL, Shih WM, Yin P (2012) Three-dimensional structures self-assembled from DNA bricks. Science 338:1177. doi: 10.1126/science.1227268

Ke Y, Sharma J, Liu M, et al (2009) Scaffolded DNA origami of a DNA tetrahedron molecular container. Nano Lett 9:2445-2447. doi: 10.1021/nl901165f

Klavžar S, Rus J (2013) Stable traces as a model for selfassembly of polypeptide nanoscale polyhedrons. MATCH Commun Math Comput Chem 70:317330.

Krishnan Y, Simmel FC (2011) Nucleic acid based molecular devices. Angew Chem Int Ed 50:31243156. doi: 10.1002/anie.200907223

Kuzyk A, Schreiber R, Fan Z, et al (2012) DNA-based selfassembly of chiral plasmonic nanostructures with tailored optical response. Nature 483:311-314. doi: $10.1038 /$ nature 10889

Li X, Yang X, Qi J, Seeman NC (1996) Antiparallel DNA double crossover molecules as components for nanoconstruction. J Am Chem Soc 118:61316140. doi: 10.1021/ja960162o

Linko V, Nummelin S, Aarnos L, et al (2016) DNA-based enzyme reactors and systems. Nanomaterials 6:139. doi: 10.3390/nano6080139
Marchi AN, Saaem I, Vogen BN, et al (2014) Toward larger DNA origami. Nano Lett 14:5740-5747. doi: $10.1021 / \mathrm{nl} 502626 \mathrm{~s}$

Mohammed A, Hajij M (2017) Unknotted strand routings of triangulated meshes. In: Proceedings, 23rd International Conference on DNA Computing and Molecular Programming.

Morse A, Adkisson W, Greene J, et al (2017) DNA origami and unknotted A-trails in torus graphs. arXiv.org

Pinheiro AV, Han D, Shih WM, Yan H (2011) Challenges and opportunities for structural DNA nanotechnology. Nat Nano 6:763-772. doi: 10.1038/nnano.2011.187

Rothemund PWK (2006a) Folding DNA to create nanoscale shapes and patterns. Nature 440:297-302. doi: $10.1038 /$ nature04586

Rothemund PWK (2006b) Scaffolded DNA origami: from generalized multicrossovers to polygonal networks. In: Chen J, Jonoska N, Rozenberg G (eds) Nanotechnology: Science and Computation. Springer Berlin Heidelberg, Berlin, Heidelberg, pp 3-21

Seeman NC (1981) Nucleic acid junctions: building blocks for genetic engineering in three dimensions. In: Sarma RH (ed) Biomolecular Stereodynamics. Adenine Press, New York, NY, pp 269-277

Seeman NC (1982) Nucleic acid junctions and lattices. J Theor Biol 99:237-247. doi: 10.1016/00225193(82)90002-9

Seeman NC (2015) Structural DNA Nanotechnology. Cambridge University Press, Cambridge, UK

Seeman NC (2010) Nanomaterials based on DNA. Annu Rev Biochem 79:65-87. doi: 10.1146/annurevbiochem-060308-102244

Seeman NC (2001) DNA nicks and nodes and nanotechnology. Nano Lett 1:22-26. doi: $10.1021 / \mathrm{nl} 000182 \mathrm{v}$

Seeman NC, Kallenbach NR (1983) Design of immobile nucleic acid junctions. Biophys J 44:201-209.

Shih WM, Quispe JD, Joyce GF (2004) A 1.7-kilobase single-stranded DNA that folds into a nanoscale octahedron. Nature 427:618-621. doi: $10.1038 /$ nature02307 
Tian Y, Wang T, Liu W, et al (2015) Prescribed nanoparticle cluster architectures and low-dimensional arrays built using octahedral DNA origami frames. Nat Nano 10:637-644.

Veneziano R, Ratanalert S, Zhang K, et al (2016) Designer nanoscale DNA assemblies programmed from the top down. Science. doi: 10.1126/science.aaf4388

Zhang C, Su M, He Y, et al (2008) Conformational flexibility facilitates self-assembly of complex DNA nanostructures. Proc Natl Acad Sci 105:1066510669. doi: $10.1073 /$ pnas. 0803841105

Zhang F, Jiang S, Wu S, et al (2015) Complex wireframe DNA origami nanostructures with multi-arm junction vertices. Nat Nano 10:779-784. doi: 10.1038/nnano.2015.162

Zhang F, Nangreave J, Liu Y, Yan H (2014) Structural DNA nanotechnology: State of the art and future perspective. J Am Chem Soc 136:11198-11211. doi: 10.1021/ja505101a

Zhang Y, Seeman NC (1994) Construction of a DNAtruncated octahedron. J Am Chem Soc 116:16611669. doi: 10.1021/ja00084a006

Zhao Z, Fu J, Dhakal S, et al (2016) Nanocaged enzymes with enhanced catalytic activity and increased stability against protease digestion. Nat Commun 7:10619. doi: 10.1038/ncomms10619 